\title{
Cefalea en urgencias
}

\section{Headache in the emergency department}

\author{
J. B. Toledo, M. Riverol, E. Martínez-Vila, P. Irimia
}

\section{RESUMEN}

Las cefaleas son un motivo común de consulta en los servicios de urgencias. La mayoría de los pacientes presentan cefaleas primarias (migraña y cefalea tensional), pero el dolor de cabeza puede ser el síntoma de una enfermedad que comprometa la vida del individuo como la hemorragia subaracnoidea. El abordaje diagnóstico del paciente con cefalea consiste en diferenciar los pacientes con cefalea primaria de los que presentan una etiología secundaria, valorar la necesidad de solicitar exploraciones complementarias y tratar adecuadamente el dolor. La anamnesis permite diferenciar las cefaleas primarias de las secundarias en la mayoría de los casos. Existen una serie de síntomas de alarma que obligan a excluir causas secundarias. En los pacientes con cefalea de inicio súbito, cuando se inicia por encima de los 50 años o en aquellos pacientes con exploración neurológica anormal, debe sospecharse una etiología secundaria.

Palabras clave. Dolor. Cefalea. Tratamientos. Emergencias.

An. Sist. Sanit. Navar. 2008; 31 (Supl. 1): 75-85.

\begin{abstract}
Headache is among the most frequent neurological symptoms in the Emergency department. Although most of the patients suffer from primary headaches (migraine), an acute headache might be the only symptom of a serious disease, such as subarachnoid haemorrhage. The physician's task is to make the diagnosis, carry out an appropriate selection of the patients who require further diagnostic evaluation and relieve the pain. An accurate history will identify most of the patients with secondary headaches. Clinicians should suspect secondary causes in sudden onset headache, headache in patients aged over 50 years, and also in those patients with abnormalities on neurological examination.
\end{abstract}

Key words. Pain. Headache. Therapeutics. Emergencies.
Departamento de Neurología. Clínica Universitaria. Universidad de Navarra. Pamplona.

\author{
Correspondencia: \\ Pablo Irimia Sieira \\ Departamento de Neurología \\ Clínica Universitaria \\ Avda. Pío XII, 36 \\ 31008 Pamplona \\ Tfno. 948255400 \\ Fax: 948296500 \\ E-mail: pirimia@unav.es
}




\section{INTRODUCCIÓN}

La cefalea supone entre el $1-4 \%$ de todas las consultas en el Servicio de Urgen$\operatorname{cias}^{1,2}$, y representa uno de los motivos más frecuentes para llamar al neurólogo de guardia ${ }^{3}$. Las cuestiones que los clínicos se plantean ante un paciente con cefalea en urgencias y que tratarán de responderse en esta revisión son tres: ¿tiene el paciente una cefalea primaria o secundaria?; ¿es necesario solicitar alguna exploración complementaria?; ¿cuál es el tratamiento de las cefaleas primarias en urgencias?

\section{¿TIENE EL PACIENTE UNA CEFALEA PRIMARIA O SECUNDARIA?}

Se definen como cefaleas primarias aquellas en las que no existe una causa estructural o metabólica que explique la sintomatología, mientras que en las secundarias el dolor de cabeza no es más que el síntoma de otra patología subyacente ${ }^{4}$. Cuando se estudia la distribución etiológica de las cefaleas de causa no traumáticas valoradas en un servicio de Urgencias ${ }^{4-6}$ las cefaleas primarias representan entre el $64-98 \%{ }^{5,6}$. Sin embargo, el objetivo fundamental del clínico que se enfrenta a un paciente con cefalea en urgencias es identificar precisamente las cefaleas secundarias que podrían comprometer la vida del paciente.

\section{Anamnesis}

En el caso de la cefalea, la historia clínica orienta el diagnóstico en la mayoría de los $\operatorname{casos}^{7,8}$. En primer lugar, debe aclararse el perfil temporal del dolor, sobre todo la forma de inicio y en concreto si la instauración es súbita o no9 . El dolor de inicio súbito orienta hacia una hemorragia subaracnoidea (HSA) y obliga a realizar exploraciones complementarias aunque la cefalea fuera de corta duración, hubiera cesado al llegar a urgencias o la exploración neurológica fuera normal. Diferentes estudios prospectivos han observado que entre los pacientes con cefalea de inicio súbito (alcanzando su máxima intensidad en menos de 1 minuto) la posibilidad de que la causa sea una HSA oscila entre el 11$25 \%$ de los $\operatorname{casos}^{3,10}$. Por otro lado, siempre debe conocerse si la cefalea se desencade- nó con la actividad física intensa, lo que también obliga a considerar la posibilidad de una HSA ${ }^{11}$.

Dentro del perfil temporal debe conocerse cuánto duran las crisis sobre todo para distinguir entre las diferentes cefaleas primarias: las crisis de migraña duran más de 4 horas sin tratamiento y la cefalea en racimos menos de 180 minutos. La duración en sí misma no permite excluir etiologías secundarias. La cefalea centinela producida por un pequeño sangrado subaracnoideo se define como una cefalea de inicio súbito y recuperación posterior que ocurre semanas o meses antes de una hemorragia subaracnoidea y que puede durar tan sólo una hora ${ }^{4,9}$. Por otro lado, las cefaleas por aumento de presión intracraneal (tumores por ejemplo) tienen un curso crónico y progresivo.

Otra de las cuestiones clave es la edad del paciente. La cefalea que se inicia después de los 50 años debe hacernos considerar la posibilidad de una arteritis temporal u otras etiologías secundarias. El inicio de crisis de migraña por encima de los 50 años es muy poco común puesto que más del $90 \%$ de los migrañosos sufren su primera crisis antes de los 40 años.

Se debe recoger también la localización, intensidad de las crisis, y una serie de síntomas o signos acompañantes como náuseas, vómitos, hipersensibilidad a la luz y el ruido, empeoramiento con la actividad física o presencia de síntomas autonómicos que permitirán clasificar adecuadamente cada tipo de cefalea (Tabla 1).

Por último, es fundamental conocer cuál ha sido la razón para que finalmente el paciente acuda al servicio de urgencias, sobre todo cuando el dolor tiene un curso crónico.

\section{Exploración física}

La exploración física debe incluir las constantes (tensión arterial y temperatura), una exploración general básica y neurológica completa. La fiebre o la presencia de un rash cutáneo debe obligar a considerar el diagnóstico de una meningitis y las tensiones arteriales superiores a 120 mm de Hg de diastólica y/o $200 \mathrm{~mm}$ de Hg de sistólica pueden provocar cefalea. 
Tabla 1. Anamnesis en el paciente con cefalea.

\begin{tabular}{|c|c|}
\hline Edad de inicio & Es un dolor de nueva aparición o una cefalea crónica \\
\hline Modo de inicio & Agudo, subagudo o crónico \\
\hline Patrón temporal & Duración y frecuencia de las crisis \\
\hline Localización del dolor & Hemicraneal, holocraneal, facial, occipital \\
\hline Síntomas acompañantes & $\begin{array}{l}\text { Náuseas, vómitos, fonofobia, fotofobia, empeoramiento } \\
\text { con la actividad física, sintomatología autonómica }\end{array}$ \\
\hline $\begin{array}{l}\text { Otros síntomas que preceden o } \\
\text { acompañan al dolor }\end{array}$ & Fotopsias, visión borrosa, pérdida de visión, parestesias \\
\hline Características del dolor & Pulsátil, opresivo, urente, punzante, descarga eléctrica \\
\hline Antecedentes de cefalea previa & Personales/familiares, similar/diferente \\
\hline Precipitantes y agravantes & Alcohol, alimentos, sueño, menstruación \\
\hline Tratamiento & $\begin{array}{l}\text { Fármacos y dosis consumidos previamente y respuesta } \\
\text { terapéutica }\end{array}$ \\
\hline Motivo de consulta en urgencias & $\begin{array}{l}\text { Motivo por el que decide acudir a urgencias en ese } \\
\text { momento concreto }\end{array}$ \\
\hline
\end{tabular}
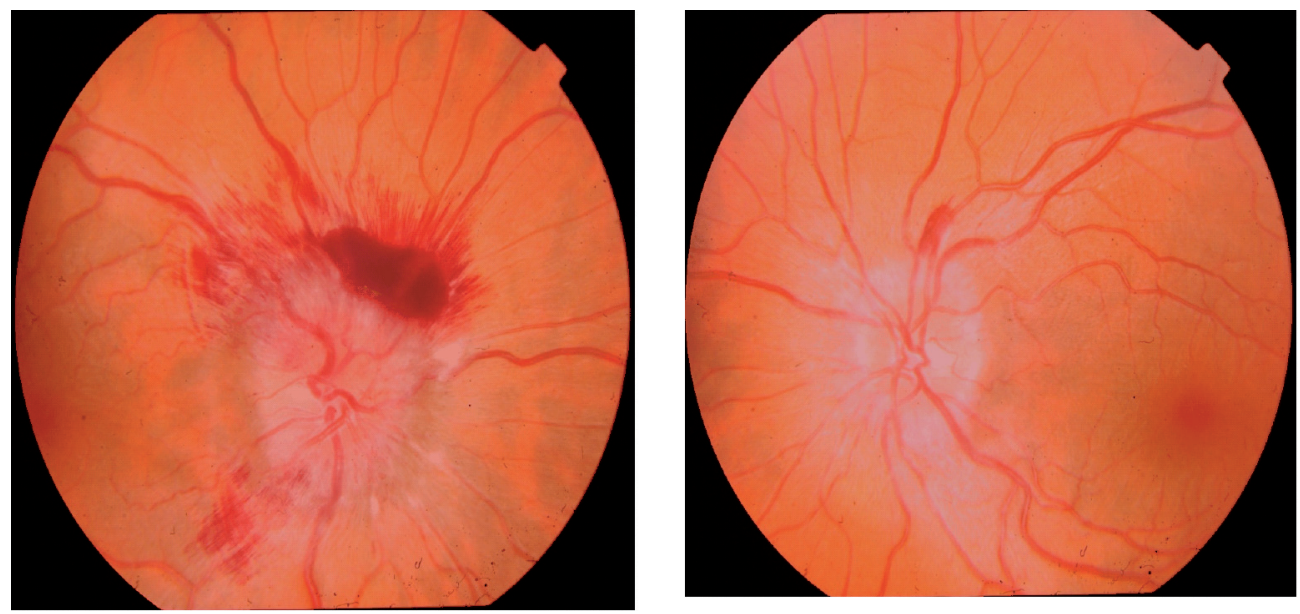

Figura 1. Fondo de ojo con papiledema bilateral. Borramiento de ambas papilas en una paciente con un tumor cerebral, apreciándose también hemorragias "en astilla".

Habrá que valorar específicamente el fondo de ojo para ver si existe un edema de papila (Fig. 1), hallazgo característico del aumento de presión intracraneal por un tumor o trombosis venosa, o signos de hemorragia subhialoidea (que típicamente se asocia a HSA). La aparición de un síndrome de Horner (ptosis y miosis ipsilaterales al dolor) es característica de la cefalea en racimos, pero también de la disección carotídea. También es imprescindible valorar la presencia de signos meníngeos, palpar las arterias temporales en pacientes mayores de 50 años (ante la sospecha de arteritis temporal), y los senos paranasales si se considera que el origen del dolor es una sinusitis. La rigidez de nuca no sólo se asocia a procesos infecciosos, sino que también es un hallazgo típico de la hemorragia subaracnoidea, la carcinomatosis meníngea o la meningitis aséptica. 


\section{Criterios "de alarma"}

Los hallazgos de la anamnesis y la exploración física detalladas van a permitir identificar los denominados criterios "de alarma" (Tabla 2) que sugieren una etiología secundaria ${ }^{10,12,13}$. Los síntomas de alarma más importantes son que el dolor se inicie de forma súbita, tras esfuerzos físicos o maniobras de Valsalva, y que tenga un carácter explosivo. Otros signos de alarma son la aparición de la cefalea en pacientes mayores de 50 años, la exploración neurológica anormal, la cefalea con sintomatología autonómica o el hecho de que el motivo de acudir a urgencias sea por otros síntomas que acompañan a la cefalea $^{10,14}$.

\section{¿ES NECESARIO SOLICITAR EXPLORACIONES COMPLEMENTARIAS?}

La decisión de realizar exploraciones complementarias está basada en la anamnesis, tal y como se muestra en el algoritmo (Fig. 2).

\section{Estudios analíticos}

En el caso de pacientes mayores de 50 años es necesario solicitar una proteína $C$ reactiva y una velocidad de sedimentación globular (VSG) para descartar una arteritis de la temporal. En menores de 50 años no suele solicitarse analítica salvo que exista sospecha de un proceso infeccioso o ante

Tabla 2. Criterios "de alarma", que sugieren una cefalea secundaria. Adaptado de Mateos y col'.

- Cefalea de comienzo súbito

- Empeoramiento reciente de una cefalea crónica

- Cefalea de frecuencia y/o intensidad creciente

- Localización unilateral estricta (excepto cefalea en racimos, hemicránea paroxística, neuralgia occipital, neuralgia del trigémino, hemicránea continua y otras cefaleas primarias unilaterales)

- Cefalea con manifestaciones acompañantes:

- Trastornos de conducta o del comportamiento

- Crisis epilépticas

- Alteración neurológica focal

- Papiledema

- Fiebre

- Náuseas y vómitos no explicables por una cefalea primaria (migraña) ni por una enfermedad sistémica

- Presencia de signos meníngeos

- Cefalea precipitada por un esfuerzo físico, tos o cambio postural

- Cefalea de características atípicas

- Cefalea refractaria a un tratamiento teóricamente correcto

- Cefalea en edades extremas de la vida

- Cefalea de presentación predominantemente nocturna

- Cefalea en pacientes oncológicos o inmunocomprometidos

Tabla 3. Indicaciones para la realización de una TC craneal en el estudio diagnóstico del paciente con cefalea.

- $\quad$ Cefalea intensa de inicio explosivo (cefalea en estallido)

- Cefalea de evolución subaguda con curso evolutivo hacia el empeoramiento progresivo

- Cefalea asociada a síntomas o signos neurológicos focales diferentes del aura migrañosa

- Cefalea asociada a papiledema o rigidez nucal

- Cefalea asociada a fiebre no explicable por enfermedad sistémica

- Cefalea y signos/síntomas de hipertensión endocraneal

- Cefalea no clasificable por la historia clínica

- Cefalea en pacientes que dudan del diagnóstico, en quienes existe una marcada ansiedad o que expresan temor ante un eventual proceso intracraneal serio 


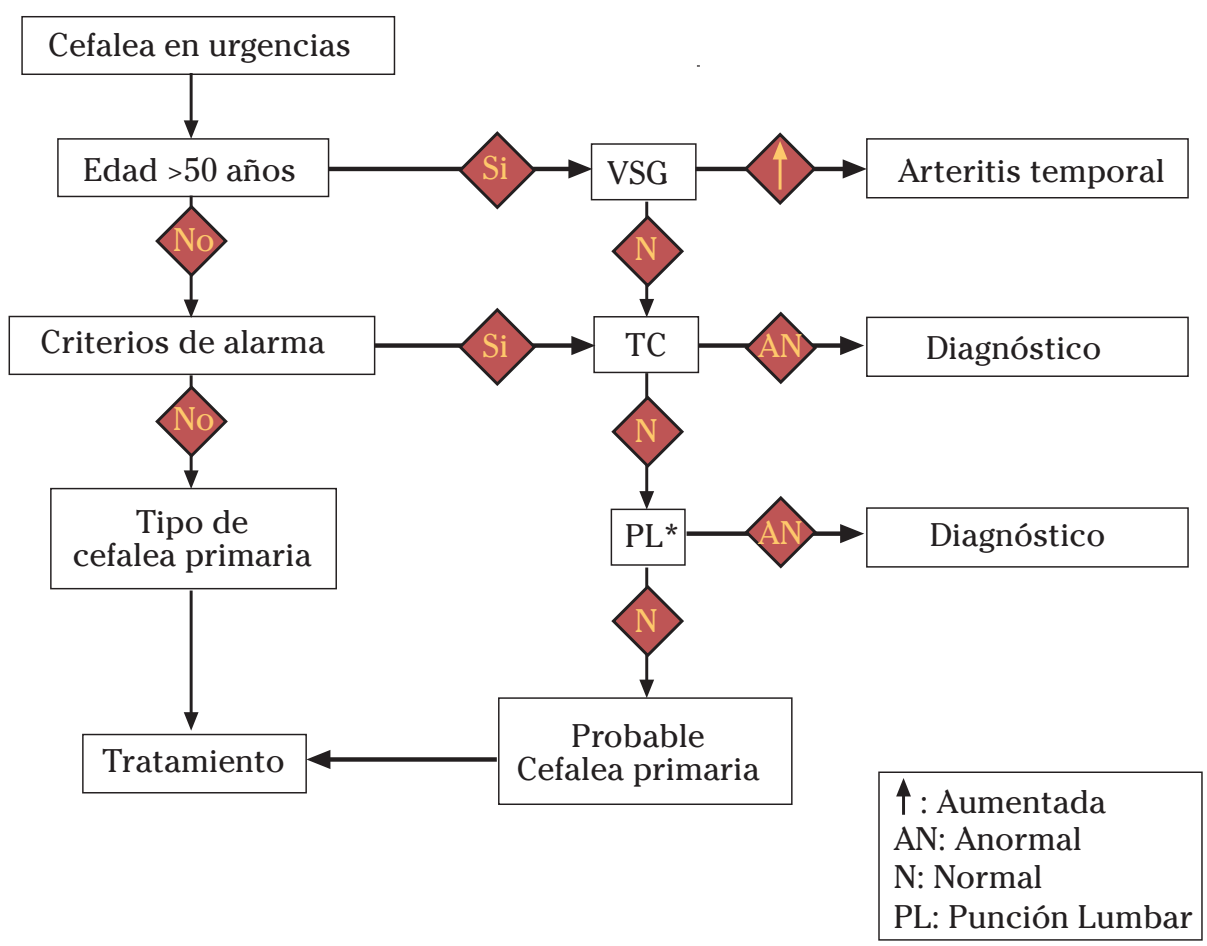

Figura 2. Algoritmo diagnóstico de la cefalea en urgencias

* El estudio de líquido cefalorraquídeo no siempre es necesario y el clínico debe decidir si debe realizarse una PL.
(Punción lumbar). TC: Tomografía computarizada. VSG: Velocidad de sedimentación globular.

una historia de vómitos repetidos y sospecha de deshidratación o alteraciones hidroelectrolíticas (y por tanto se solicitará al menos ionograma y función renal).

\section{Neuroimagen}

La decisión de realizar una prueba de neuroimagen debe sustentarse en la historia clínica. Existen una serie de indicaciones claras (Tabla 3) sobre todo cuando existen datos clínicos o hallazgos en la exploración que sugieren una cefalea secundaria, cuando las características del dolor no se ajustan a una cefalea primaria y sobre todo en presencia de criterios de alarma ${ }^{11,14-16}$. La radiología simple está indi- cada en caso de sospecha de mastoiditis o sinusitis. Sin embargo, para valoración del parénquima cerebral la exploración de elección en urgencias es una tomografía computarizada (TC) cerebral, inicialmente sin contraste. La sensibilidad y especificidad de la TC cerebral para el diagnóstico de HSA (Fig. 3) son superiores al 98\% ${ }^{17}$. Debe recordarse que la TC tiene menor rendimiento en caso de lesiones de fosa posterior, silla turca, seno cavernoso y para descartar malformaciones de la charnela occipito-vertebral (anomalía de Chiari), o en algunas entidades específicas que producen cefalea como las secundarias a hipotensión licuoral, o por una trombosis 


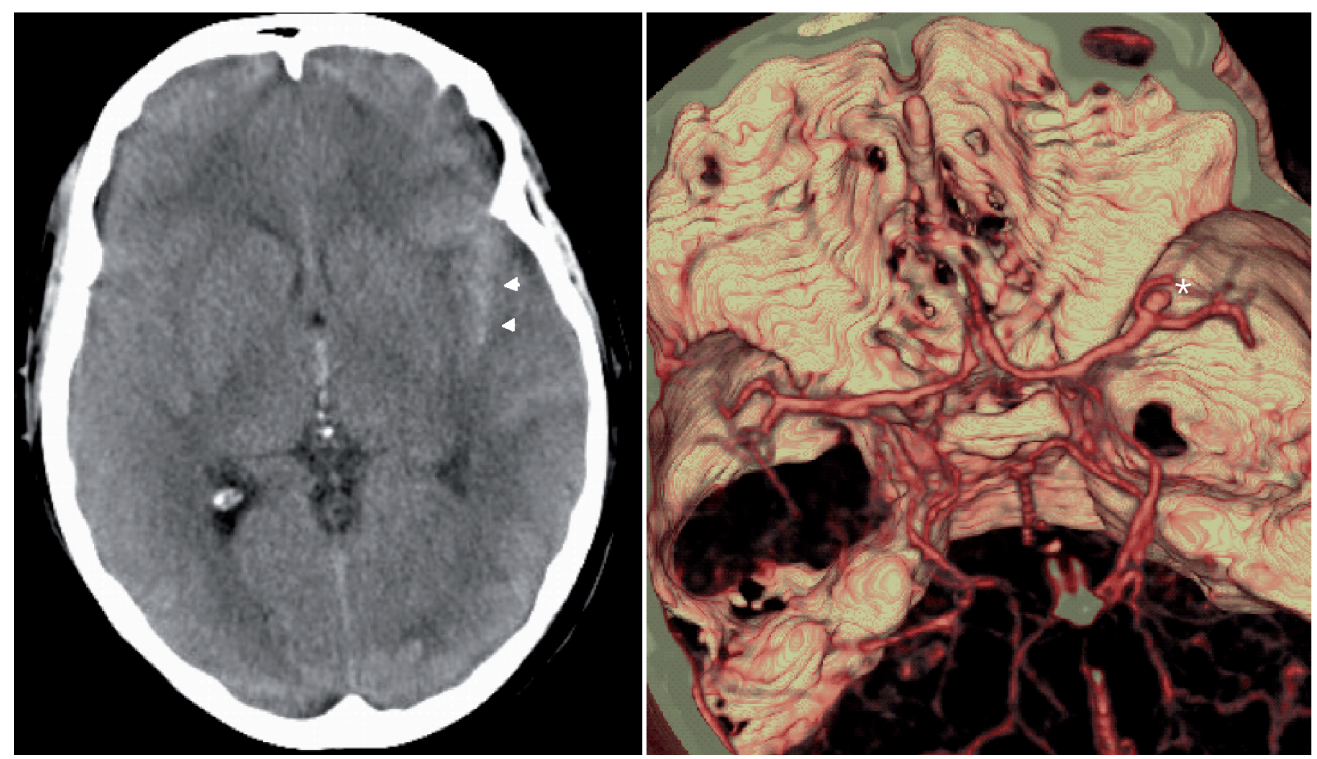

Figura 3. A) TC cerebral sin contraste: Hemorragia subaracnoidea que afecta a cisura silviana izquierda (puntas de flecha). B) Angio-TC: aneurisma en bifurcación de arteria silviana izquierda (asterisco).

venosa cerebral. En estos casos estaría indicado realizar una resonancia magnética cerebral.

\section{Estudio de líquido cefalorraquídeo}

En caso de sospecha de meningitis, encefalitis o HSA con TC normal está indicado realizar una punción lumbar. En general, debe realizarse una TC cerebral antes de la realización de una punción lumbar por el riesgo de herniación (y muerte) asociado a la presencia de lesiones intracraneales ocupantes de espacio. En pacientes con un absceso cerebral no está indicado el estudio licuoral, porque en la mayoría de los casos no permitirá filiar el germen responsable del cuadro. Ante la sospecha de hemorragia subaracnoidea se recomienda realizar la punción lumbar cuando hayan transcurrido 12 horas desde el inicio de la sintomatología para determinar la xantocromía mediante técnicas de espectrometría $^{18,19}$. Si la sospecha es de hipertensión intracraneal, tras realizar una TC cere- bral habría que medir la presión de líquido cefalorraquídeo.

\section{¿CUÁL ES EL TRATAMIENTO DE LAS CEFALEAS PRIMARIAS EN URGENCIAS?}

\section{Migraña}

La migraña representa un $37-48 \%$ de los pacientes que acuden a urgencias por cefalea. Se caracteriza por una cefalea de instauración progresiva, que dura entre 4 y 72 horas; suele acompañarse de náuseas, vómitos, fonofobia y fotofobia, y característicamente empeora con la actividad física (Tabla 4). En general, los pacientes han sufrido crisis similares previamente y el motivo de acudir a urgencias es que el dolor no ha respondido a su tratamiento habitual. De acuerdo con la Clasificación Internacional de Cefaleas se distinguen las crisis agudas de migraña (de 72 horas de evolución) del denominado estatus migrañoso en el que el dolor dura más de 72 horas y es muy incapacitante ${ }^{4}$. 
Tabla 4. Criterios diagnósticos de migraña4.

A. Al menos cinco crisis cumpliendo los criterios B, C y D

B. Cefalea que dura entre 4 y 72 horas (no tratada o con tratamiento ineficaz)

C. Cefalea que presenta, al menos, dos de las siguientes características:

1. Localización unilateral

2. Cualidad pulsátil

3. Intensidad moderada a severa

4. Se agrava por o conduce a evitar la actividad física (e: caminar o subir escaleras)

D. Durante el dolor presenta, al menos, uno de los siguientes síntomas:

1. Náuseas y/o vómitos

2. Fotofobia y fonofobia

E. No es atribuible a otra causa

Ante una crisis aguda de migraña debe valorarse en primer lugar si el paciente tolera medicación por vía oral. En este caso se puede administrar un triptán vía oral. Sin embargo, si no hay posibilidad de administrar fármacos por vía oral está indicado el tratamiento mediante triptanes por vía subcutánea (sumatriptán) o inhalada (sumatriptán o zolmitriptán). Debe recordarse que los triptanes no deben administrase en caso de que exista una toma reciente de ergóticos $\mathrm{u}$ otros triptanes, y que están contraindicados en pacientes con cardiopatía isquémica, claudicación intermitente e hipertensión arterial no controlada.

Los antiinflamatorios no esteroideos (AINES) por vía intramuscular (im) o intravenosa (iv) son probablemente los fármacos que se prescriben con mayor frecuencia. En el caso de la migraña el diclofenaco 75 mg o ketorolaco $30-60$ mg $^{20,21}$ son los más utilizados.

En caso de que el paciente presente náuseas y vómitos podría administrarse metoclopramida, asociada con AINES para el dolor ${ }^{22-25}$. En ocasiones, tan solo con hidratar adecuadamente al paciente la sintomatología mejora y diferentes ensayos clínicos han demostrado que la metoclopramida tiene efecto analgésico per se en el caso de la migraña ${ }^{26-28}$. Si el paciente tiene mucha ansiedad o importantes trastornos del sueño deberá valorarse el uso de benzodiacepinas, como el diazepam a dosis bajas (5-10 mg) que con frecuencia se administran de forma combinada con AINES por vía im (diclofenaco y diazepam $5 \mathrm{mg}$ ). La clorpromazina tiene también efecto analgésico, y se ha utilizado en pacientes con migraña de larga duración o estatus migrañoso. Puede administrarse por vía iv o im $(0,1 \mathrm{mg} / \mathrm{kg}$ hasta 37,5 $\mathrm{mg})^{27,31}$. Entre sus efectos secundarios se encuentran la somnolencia y la hipotensión arterial, por lo que cuando se administra por vía iv se recomienda administrar previamente $500 \mathrm{ml}$ de suero fisiológico e infundir la medicación de forma lenta, en al menos 15 minutos. Otro neuroléptico estudiado en migraña ha sido el haloperidol, que es efectivo, pero presenta importantes efectos secundarios, por lo que no se recomienda como primera elección ${ }^{32}$.

Los opiáceos no deberían usarse de forma rutinaria, por el riesgo de dependencia, sedación (y depresión respiratoria) y por la posibilidad de que aumenten las náuseas y vómitos. El fármaco más utilizado de este grupo es la meperidina en dosis de 50-100 mg im o subcutánea ${ }^{33-34}$.

En caso de estatus migrañoso, o en migrañas de larga duración podrían utilizarse esteroides como la dexametasona o metilprednisolona. Aunque no existen ensayos clínicos que avalen su eficacia la mayoría de los expertos consideran que la dexametasona es el tratamiento de elección en estatus migrañoso. La dosis no está bien establecida y se han utilizado entre 4-20 $\mathrm{mg}$ en bolus. Lo habitual es administrar una dosis media, entre 12-16 mg. La metilprednisolona puede administrarse durante el embarazo.

Otra posibilidad de tratamiento en urgencias es el ácido valproico. Las dosis que se han utilizado son ligeramente inferiores a las que se utilizan en el estatus 
convulsivo, y se administran mediante una infusión rápida ${ }^{35-40}$.

Por tanto, lo recomendable en una crisis de migraña de larga duración o un estatus migrañoso es utilizar sumatriptán subcutáneo o bien la combinación de AINES con metoclopramida. Si la cefalea no mejora el siguiente paso sería la administración de esteroides. En caso de persistir el dolor habría que replantearse si el diagnóstico es correcto y si se considera que se trata de una migraña administrar clorpromacina o valproato (Fig. 4).

\section{Cefaleas trigémino autonómicas: cefalea en racimos}

La cefalea trigémino autonómica más frecuente es la cefalea en racimos. Los pacientes presentan crisis de corta dura- ción (en general entre 30 minutos y 3 horas), que se acompañan de sintomatología autonómica ipsilateral al dolor (lagrimeo, rinorrea, inyección conjuntival y/o miosis) y una gran inquietud. Los pacientes con cefalea en racimos, al contrario de lo que ocurre en la migraña, no pueden permanecer en reposo absoluto. Para las crisis agudas está indicado el tratamiento con sumatriptán subcutáneo, sumatriptán o zomitriptán inhalados, o bien el uso de oxígeno $^{41}$. El oxígeno es eficaz en aproximadamente el $80 \%$ de los pacientes. Debe utilizarse oxígeno inhalado a más de 9 litros por minuto para que sea efectivo. Generalmente se recomienda utilizar el oxígeno a más de 12 litros por minuto durante las crisis. Las mascarillas utilizadas habitualmente para administrar oxígeno tienen una serie de agujeros a ambos lados que deben ser

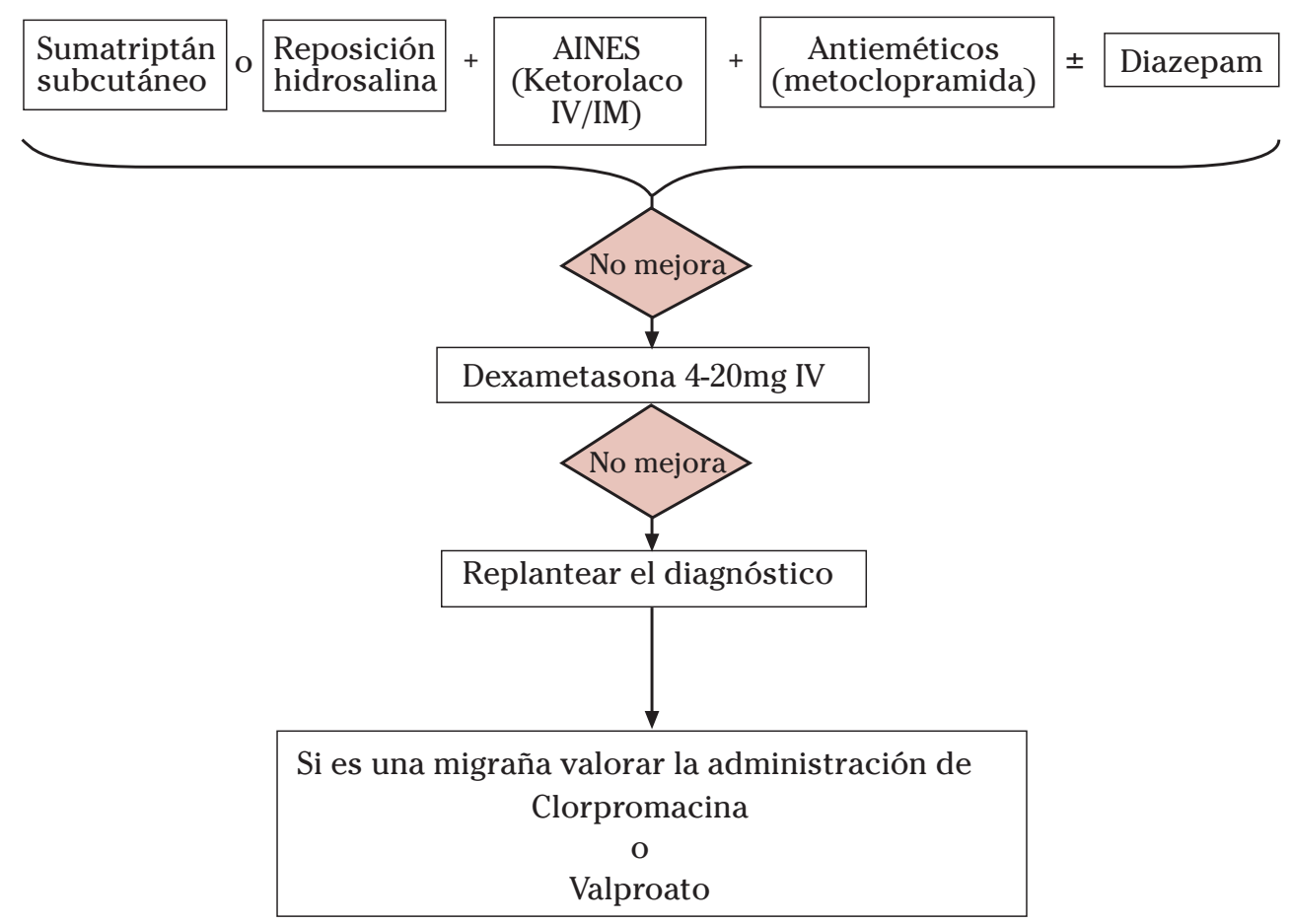

Figura 4. Algoritmo de tratamiento de la migraña de larga duración y estatus migrañoso. Adaptado de Mateos y $\mathrm{col}^{8}$. 
tapados, para incrementar la eficacia del tratamiento, o bien utilizar mascarilla con reservorio. Aunque el tiempo necesario para que el oxígeno sea efectivo es variable, en general se recomienda utilizarlo durante unos 10-15 minutos.

Es recomendable derivar a pacientes con cefalea en racimos a una consulta especializada para establecer el tratamiento preventivo más adecuado y se recomienda solicitar un estudio de neuroimagen, preferentemente resonancia magnética. Si el diagnóstico de cefalea en racimos es firme podría iniciarse el tratamiento preventivo a corto plazo con prednisona oral a dosis de $1 \mathrm{mg} / \mathrm{kg} /$ día por la mañana (máximo $80 \mathrm{mg}$ ) durante 5 días con una pauta descendente ${ }^{42}$.

\section{CEFALEAS SECUNDARIAS}

La constelación de fiebre, malestar general, cefalea y rinorrea puruleta son características de sinusitis aguda. Característicamente, la intensidad del dolor aumenta al inclinarse hacia delante y su localización varía en función del seno implicado, siendo frontal en caso del seno frontal, antral con irradiación frontal en caso de los senos maxilares, retroorbitario y línea media en la etmoiditis y en el caso esfenoidal puede ser tanto posterior como anterior $^{43}$.

La hipertensión arterial de nueva aparición puede ser una causa de cefalea y en las embarazadas debe realizarse una medición de tensión arterial ante la aparición de cefalea.

La presencia de una VSG elevada (mayor de 50) en pacientes mayores de 50 años, debe hacernos sospechar una arteritis de la temporal, que suele cursar con cefalea hemicraneal de predominio frontotemporal, claudicación mandibular, síntomas constitucionales (astenia, anorexia, sudoración) y alteraciones de la visión que pueden derivar en ceguera permanente si la corticoterapia no se inicia precozmente. En la exploración puede presentar endurecimiento de la arteria, ausencia de pulso palpable y dolor a la palpación. Debe iniciarse tratamiento con prednisona $1 \mathrm{mg} / \mathrm{kg} / \mathrm{día}$ con posterior pauta descendente.
El glaucoma de ángulo cerrado presenta un intenso dolor en ojo y órbita y suele asociar síntomas vegetativos con importante pérdida de agudeza visual unilateral. A la exploración se observa ojo rojo, pupila media arreactiva y un aumento de la presión intraocular.

Si a la cefalea se le añade dolor cervical, debe incluirse en el diagnóstico diferencial la posibilidad de una disección arterial. En el caso de afectarse la arteria carótida cervical puede sumarse al dolor la presencia de síndrome de Horner, tinnitus pulsátil, afectación de pares craneales bajos, amaurosis y fenómenos isquémicos en el territorio de la arteria cerebral media $^{44}$. En el caso de la disección de arterias vertebrales, además del dolor, puede aparecer sensación de mareo y otra sintomatología inducida por la isquemia en territorio de la arteria cerebelosa posterioinferior ${ }^{44}$.

Existen también cefaleas inducidas por determinadas medicaciones como nifedipino, nitroglicerina, dipiridamol o sildenafilo ${ }^{45}$.

En ocasiones, la cefalea puede ser un síntoma acompañante o el único síntoma de una isquemia miocárdica. Típicamente esta cefalea se desencadena por el ejercicio y desaparece en reposo o tras la administración de nitroglicerina sublingual ${ }^{46}$.

\section{BIBLIOGRAFÍA}

1. Newman LC, Lipton RB. Emergency department evaluation of headache. Neurol Clin 1998; 16: 285-303.

2. Morgenstern LB, Huber JC, Luna-Gonzales H, SALDin KR, GRotTA JC, SHAW SG et al. Headache in the emergency department. Headache 2001; 41: 537-541.

3. Jimenez-CABallero PE, Marsal-Alonso C, Alvarez-Tejerina A. Análisis de la actividad asistencial en las guardias de neurología y su repercusión en el hospital. Rev Neurol 2004; 39: 120-124.

4. The International Classification of Headache Disorders: 2nd edition. Cephalalgia 2004; 24 Suppl 1: 9-160.

5. Friedman, BW, Hochberg ML, Esser D, Grosberg B, Corbo J, Toosi B et al. Applying the International Classification of Headache Disorders to the Emergency Department: An Assessment of Reproducibility and the Frequency With Which a Unique Diagnosis 
Can Be Assigned to Every Acute Headache Presentation. Ann Emerg Med 2007; 49: 409419.

6. Goldstein JN, Camargo Ca Jr, Pelletier AJ, EDLow JA. Headache in United States emergency departments: demographics, work-up and frequency of pathological diagnoses. Cephalalgia 2006; 26: 684-690.

7. EzPELETA D. Diagnóstico diferencial de la cefalea en urgencias Jano: Medicina y Humanidades 2007; 1662: 31-35.

8. Actitud diagnóstica y teapéutica en la cefalea: Recomendaciones 2006. Grupo de estudio de cefaleas de la Sociedad Española de Neurología. Madrid: Ergón 2006.

9. DAVEnPort R. Acute headache in the emergency department. J Neurol Neurosurg Psychiatry 2002; 72 Suppl 2: p. ii33-ii37.

10. Locker TE, Thompson C, Rylance J, Mason Sm. The utility of clinical features in patients presenting with nontraumatic headache: an investigation of adult patients attending an emergency department. Headache 2006; 46 : 954-961.

11. Landtblom AM, Fridiksson S, Boivie J, Hillman J, Johansson G, Johansson I. Sudden onset headache: a prospective study of features, incidence and causes. Cephalalgia 2002; 22: 354-360.

12. Cortelli P, Cevoli S, Nonino F, Baronciani D, MAgRini N, RE G et al. Evidence-based diagnosis of nontraumatic headache in the emergency department: a consensus statement on four clinical scenarios. Headache 2004; 44: 587-595.

13. Newman LC, Lipton RB. Emergency department evaluation of headaches. Neurol Clin 1998; 16: 285-303.

14. Detsky ME, McDonald DR, Baerlocher MO, Tomlison GA, McCrory DC, Booth CM. Does this patient with headache have a migraine or need neuroimaging? JAMA 2006; 296 : 1274-1283.

15. Sempere AP, Porta-Etessam J, Medrano V, García-Morales I, Concepción L, Ramos A. Neuroimaging in the evaluation of patients with non-acute headache. Cephalalgia 2005; 25: 30-35.

16. Duarte J, Sempere AP, Delgado JA, Naranjo G, Sevillano MD, Clavería LE. Headache of recent onset in adults: a prospective population-based study. Acta Neurol Scand 1996; 94: 67-70.

17. Masdeu JC, IRimia $\mathrm{P}$, Asenbaum $\mathrm{S}$, Bogousslavsky J, BRainin M, Chabriat $\mathrm{H}$ et al. EFNS guideline on neuroimaging in acute stroke. Report of an EFNS task force. Eur J Neurol 2006; 13: 1271-1283.

18. National guidelines for analysis of cerebrospinal fluid for bilirubin in suspected subarachnoid haemorrhage. Ann Clin Biochem 2003; 40: 481-488.

19. Vermeulen M, Hasan D, Blijenberg BG, Hijdra A, VAN GIJM J. Xanthochromia after subarachnoid haemorrhage needs no revisitation. J Neurol Neurosurg Psychiatry 1989; 52: 826-828.

20. Karachalios GN, Fotiadou A, Chrisikos N, Karabetson A, Kehagioglou $K$. et al. Treatment of acute migraine attack with diclofenac sodium: a double-blind study. Headache 1992; 32: 98-100.

21. Hering R, Couturier EG, Steiner TJ. Intramuscular diclofenac in late treatment of migraine. Cephalalgia 1996; 16: 453.

22. Acute treatment of migraine attacks: efficacy and safety of a nonsteroidal antiinflammatory drug, diclofenac-potassium, in comparison to oral sumatriptan and placebo. The Diclofenac-K/Sumatriptan Migraine Study Group. Cephalalgia 1999; 19: 232-240.

23. Seim MB, MARCh JA, Dunn KA. Intravenous ketorolac vs intravenous prochlorperazine for the treatment of migraine headaches. Acad Emerg Med 1998; 5: 573-576.

24. Matchar DB, Young WB, Rosenerg JH, Pietrzak MP, Silberstein SD, Lipton RB et al. Multispecialty consensus on diagnosis and treatment of headache: pharmacological management of acute attacks. http://www.aan.com/professionals/practic e/pdfs/gl0087.pdf., 2004.

25. Engindeniz Z, Demircan C, Karli N, Armagan E, Bulut M, Aydin $\mathrm{T}$ et al. Intramuscular tramadol vs. diclofenac sodium for the treatment of acute migraine attacks in emergency department: a prospective, randomised, double-blind study. J Head Pain 2005; 6: 143-148.

26. Bell R, Montoya D, Shuaib A, Lee MA. A comparative trial of three agents in the treatment of acute migraine headache. Ann Emerg Med 1990; 19: 1079-1082.

27. Cameron JD, Lane PL, Speechley M. Intravenous chlorpromazine vs intravenous metoclopramide in acute migraine headache. Acad Emerg Med 1995; 2: 597-602.

28. Friedman BW, Corbo J, Lipton RB, BiJur PE, Esses D, Solorzano C et al. A trial of metoclopramide vs sumatriptan for the 
emergency department treatment of migraines. Neurology 2005; 64: 463-468.

29. McEwen JI, O'Connor HM, Dinsdale HB. Treatment of migraine with intramuscular chlorpromazine. Ann Emerg Med 1987; 16: 758-763.

30. Bigal ME, Bordini CA, Speciali JG. Intravenous chlorpromazine in the emergency department treatment of migraines: a randomized controlled trial. J Emerg Med 2002; 23: 141-148.

31. Shrestha M, Singh R, Moreden J, Hayes JE. Ketorolac vs chlorpromazine in the treatment of acute migraine without aura. A prospective, randomized, double-blind trial. Arch Intern Med 1996; 156: 1725-1728.

32. Honkaniemi J, Limatainen S, Rainesalo S, SulaVNori S. Haloperidol in the acute treatment of migraine: a randomized, double-blind, placebo-controlled study. Headache 2006; 46: 781-787.

33. Klapper JA, Stanton J. Current emergency treatment of severe migraine headaches. Headache 1993; 33: 560-562.

34. Lane PL, Mclellan BA, Baggoley CJ. Comparative efficacy of chlorpromazine and meperidine with dimenhydrinate in migraine headache. Ann Emerg Med 1989; 18: 360-365.

35. Friedman BW, Greenwald P, Bania TC, Esses D, Hochberg M, Solorzano C et al. Randomized trial of IV dexamethasone for acute migraine in the emergency department. Neurology 2007; 69: 2038-2044.

36. Donaldson D, Sundermann R, JaCKSON R, BASTANI A. Intravenous dexamethasone vs placebo as adjunctive therapy to reduce the recurrence rate of acute migraine headaches: a multicenter, double-blinded, placebo-controlled randomized clinical trial. Am J Emerg Med 2008; 26: 124-130.

37. Kelly AM, KerR D, Clooney M. Impact of oral dexamethasone versus placebo after ED treatment of migraine with phenothiazines on the rate of recurrent headache: a randomised controlled trial. Emerg Med J 2008; 25: 26-29.

38. Tanen DA, Miller S, French T, Riffenburgh $\mathrm{RH}$. Intravenous sodium valproate versus prochlorperazine for the emergency department treatment of acute migraine headaches: a prospective, randomized, double-blind trial. Ann Emerg Med 2003; 41: 847-853.

39. Stillman MJ, ZaJac D, Rybicki LA. Treatment of primary headache disorders with intravenous valproate: initial outpatient experience. Headache 2004; 44: 65-69.

40. GREEN MW. The emergency management of headaches. Neurologist 2003; 9: 93-98.

41. Cohen AS, Matharu MS, Goadsby PJ. Trigeminal autonomic cephalalgias: current and future treatments. Headache 2007; 47: 969-980.

42. Antonaci F, Costa A, Candeloro E, Siahistad O, NAPPI G. Single high-dose steroid treatment in episodic cluster headache. Cephalalgia 2005; 25: 290-295.

43. Levine HL, Setzen M, Cady RK, Dodick DN, Schreiber CP, ERoss EJ et al. An otolaryngology, neurology, allergy, and primary care consensus on diagnosis and treatment of sinus headache. Otolaryngol Head Neck Surg 2006; 134: 516-523.

44. CAPLAN LR. Dissections of brain-supplying arteries. Nat Clin Pract Neurol 2008; 4: 3442.

45. Young WB. Drug-induced headache. Neurol Clin 2004; 22: 173-184.

46. Gutiérrez Morlote J, Fernández García J, Timiraos Fernández JJ, Llano Catedral M, Rodríguez Rodríguez E, Pascual Gómez J. Cefalea cardíaca: ¿Una entidad infradiagnosticada? Rev Esp Cardiol 2005; 58: 1476-1468. 
